\title{
AVALIAÇÃO DAS DIFICULDADES DOS ESTUDANTES DE GRADUAÇÃO NA ELABORAÇÃO DE RELATÓRIOS DE QUÍMICA EXPERIMENTAL
}

\author{
Klenicy K. L. Yamaguchi ${ }^{\mathrm{a}, *,(1)}$, Hudinilson Kendy de Lima Yamaguchi ${ }^{\mathrm{b}}$ e Jath da Silva e Silva ${ }^{\mathrm{c}}$ \\ ${ }^{a}$ Colegiado de Ciências: Biologia e Química, Instituto de Saúde e Biotecnologia, Universidade Federal do Amazonas, 69460-000 \\ Coari - AM, Brasil \\ 'Instituto Federal do Amazonas, campus Coari, 69460-000, Coari - AM, Brasil \\ ${ }^{\circ}$ Coordenação acadêmica, Instituto de Saúde e Biotecnologia, Universidade Federal do Amazonas, 69460-000 Coari - AM, Brasil
}

Recebido em 04/09/2019; aceito em 15/01/2020; publicado na web em 31/03/2020

\begin{abstract}
EVALUATING OF DIFFICULTIES OF UNDERGRADUATE ON THE REPORT OF CHEMISTRY LAB EXPERIMENTAL. Scientific reports are important tools for show results of experimental activities undertaken at undergraduate level. The aims of this research were detecting difficulties of undergraduate students in the elaboration of reports in the experimental chemistry subjects of students of the Science: Biology and Chemistry course at the Federal University of Amazonas in different periods $\left(2^{\text {nd }}\right.$ to $\left.10^{\text {th }}\right)$. Results and discussion and theoretical bibliographic were topics reported with the most difficulty. The students showed little reading habit, however, which increases with the time they spend in university, also reflecting the retention rate in the experimental subjects. These results point to possibilities to reduce the difficulties in the elaboration of experimental reports by intervention alternatives more appropriate to the desired learning process, based on the students' reality. Detecting these points becomes an important to assist them in the learning process of academic writing.
\end{abstract}

Keywords: scientific communication; writing; chemistry education; Amazon.

\section{INTRODUÇÃO}

A química é um dos mais importantes ramos da ciência, permitindo a compreensão do que acontece no universo por meio das propriedades e mudanças da matéria. ${ }^{1}$ As práticas laboratoriais dessa disciplina favorecem a construção do conhecimento, alicerçando a teoria com a realidade empírica e contribuindo para a consolidação de um conhecimento acadêmico, profissional e social mais enriquecido e fundamentado em cada um dos participantes. ${ }^{2} \mathrm{Um}$ dos principais instrumentos utilizados para mensurar o aprendizado adquirido nas aulas práticas experimentais é o relatório das aulas de laboratório (relatório experimental), onde os alunos sistematizam o aprendizado na descrição, análise e discussão das observações da prática realizada.

Os relatórios das atividades experimentais, somados as observações na sala de aula, questionamentos e análise crítica-reflexiva, contribuem para o rompimento da visão dogmática da Ciência, trazendo a materialização de conceitos que muitas vezes são encarados de uma forma abstrata pelos estudantes. ${ }^{2,3} \mathrm{O}$ conhecimento expresso pelos alunos na discussão da atividade experimental pode proporcionar o início de um novo ciclo de aprendizagem, auxiliando o professor a mapear os conhecimentos do grupo sobre o tema estudado e avaliar de forma individual o crescimento dos acadêmicos. ${ }^{4}$ Além disso, verifica-se que o aprendizado compreende a apropriação de descrever, comparar, classificar, analisar, discutir, teorizar, concluir e generalizar esses aspectos podem ser expressos tanto na forma oral quanto da escrita. ${ }^{5}$

Segundo Oliveira e Queiroz, ${ }^{6}$ a capacidade de comunicação em linguagem científica é uma habilidade valiosa para aqueles que se dedicam à produção do conhecimento científico. No entanto, o que se tem observado nas pesquisas sobre a temática, é que estão sendo frequentes os relatos sobre a dificuldade encontrada por alunos de graduação na comunicação de seus conhecimentos. ${ }^{6-8}$

\footnotetext{
*e-mail: klenicy@gmail.com
}

Estudos apontam que essa flagrante dificuldade engloba desde aspectos relativos à articulação de ideias até aqueles de ordem meramente estrutural e/ou formal. ${ }^{7,9,10}$ Entre as dificuldades reportadas, tem-se a complexidade da linguagem, pouca interpretação de texto, coleta de informações em fontes bibliográficas inadequadas, entre outros pontos que formam um conjunto de situações que comprometem o desenvolvimento acadêmico e que podem persistir e se estender para a vida profissional. ${ }^{11}$

As dificuldades dos estudantes de graduação em comunicar-se por meio da escrita, ou através do entendimento sobre os conteúdos do curso de forma adequada, ou até mesmo de apresentar a compreensão dos fenômenos científicos obtidos nas aulas experimentais há muito tempo, é considerada um problema. Várias pesquisas de âmbito nacional e internacional têm abordado de forma consistente e satisfatória experiências relacionadas ao aprimoramento desse tipo de comunicação em cursos universitários, como o uso de reflexões, análises investigativas e uso de artigos científicos e outros gêneros textuais. ${ }^{11-14}$ Porém, pouco é descrito sobre a detecção dessas dificuldades, que é um dos pontos principais para a busca da solução desse entrave.

O uso da comunicação científica é essencial para a aprendizagem, bem como para o desenvolvimento da Ciência, no entanto, apesar da importância dos processos de comunicação e da linguagem científica no desenvolvimento da ciência, esse é um aspecto pouco abordado durante o período de graduação e a atenção dada é insuficiente. Usualmente esse tema é enfatizado somente nos programas de pósgraduação devido às exigências dos órgãos avaliadores de produção. ${ }^{5}$

A dificuldade entre os alunos em expor o aprendizado por meio dessa ferramenta metodológica tem como consequência a elaboração de produtos de baixa qualidade que nem sempre conseguem revelar um entendimento completo da disciplina, e que pode desestimular os estudantes. ${ }^{15}$ Verifica-se, dessa forma, a necessidade de pesquisas que forneçam subsídios que auxiliem na compreensão das dificuldades que os acadêmicos têm em relação à elaboração da escrita científica de relatórios experimentais. Para tanto, neste trabalho buscou-se 
mapear e discutir sobre as dificuldades enfrentadas na elaboração de relatórios de aulas experimentais de Química de acadêmicos do curso de Ciências: Biologia e Química da Universidade Federal do Amazonas - UFAM. Este artigo visa contribuir com questões sobre os pontos acadêmicos que devem ser considerados relevantes para a busca do aprimoramento da comunicação científica e que possam favorecer/contribuir com a produção de uma escrita de qualidade no ensino de química.

\section{METODOLOGIA}

A metodologia utilizada classifica-se como explicativa, tendo como objetivo básico a identificação dos fatores que determinam ou que contribuem para a ocorrência da dificuldade na elaboração dos relatórios experimentais. ${ }^{16}$ Apresenta a perspectiva de pesquisa qualitativa e quantitativa, centrada na autoanálise dos discentes e análise de dados secundários. As informações utilizadas na pesquisa de campo foram obtidas por meio de questionários aplicados aos acadêmicos do Instituto de Saúde e Biotecnologia - ISB da UFAM, no município de Coari, Amazonas, Brasil, caracterizando o trabalho como um estudo de caso.

O critério adotado para seleção dos alunos foi por meio do aceite em participar da pesquisa e de terem cursado alguma disciplina de Química experimental, a saber: Química Geral, Química Orgânica, Química Inorgânica, Química Analítica e Físico-Química, durante o referido curso de graduação. Para tanto, foram convidados a participar os discentes de todas as turmas do curso de Ciências: Biologia e Química ( $2^{\circ}$ ao $10^{\circ}$ período), que estivessem cursando ou que já cursaram as disciplinas citadas. A amostra se consistiu em 83 indivíduos que, após assinarem o termo de consentimento livre e esclarecido, receberam um questionário contendo 15 perguntas.

A segunda etapa do percurso metodológico deu-se por meio da coleta de dados institucionais (dados secundários), relativos à situação acadêmica dos alunos que já haviam cursado as disciplinas citadas anteriormente, relacionando o percentual de aprovação com o de retenção, além da natureza deste último. A retenção foi calculada baseando-se na resolução vigente, considerando em retenção os estudantes que reprovaram por falta, trancamento ou nota inferior à média 5,0. Esses dados institucionais relatam as informações referentes ao período de 2007 a 2019, ou seja, os dados desde a criação do instituto.

Posteriormente, os resultados foram agrupados e organizados em categorias. Os valores foram tabulados e analisados quantitativamente de modo a apresentá-los sob a forma de percentual e de número absoluto. A análise de dados deu-se por meio da proporção entre o número de indivíduos que forneceram cada resposta e o total de pessoas que responderam às perguntas. Além disso, também se pôde aplicar, sobre o material coletado, uma análise qualitativa das dificuldades.

Os dados obtidos foram submetidos à análise estatística calculando a média, moda e mediana. Para análise descritiva dos resultados, foram utilizados gráficos e tabelas a fim de permitir uma observação mais clara dos dados coletados. Utilizou-se, para tanto, o programa de estatística R $2.14 .0^{\circledR}$ para Windows.

\section{RESULTADOS E DISCUSSÃO}

Os relatórios de atividades experimentais apresentam-se como o principal método de avaliação para os estudantes que cursam as disciplinas de Química experimental, apresentando o entendimento e a compreensão acerca dos resultados das análises e reações químicas ocorridas nas aulas. Nesse trabalho foi possível investigar as percepções dos alunos no que diz respeito à elaboração de relatórios de aulas experimentais. Para tanto, os resultados foram formados nas seguintes etapas: a) caracterização de perfis dos discentes; b) detecção da dificuldade; c) identificação dos dados institucionais referentes às disciplinas experimentais; e d) apresentação de estratégias de ações voltadas à possíveis intervenções.

\section{Caracterização dos sujeitos}

O processo de análise dos dados iniciou-se com a apreciação dos questionários. Verifica-se que as características dos sujeitos são importantes para avaliar a perspectiva em que a pesquisa está inserida. Na pesquisa realizada, os 83 acadêmicos estavam devidamente matriculados no curso de Ciências: Biologia e Química, sendo que $36 \%$ estavam no segundo período; $20 \%$ quarto; $24 \%$ no sexto; $16 \%$ no oitavo e $4 \%$ eram finalistas e estavam no décimo período (Figura 1A). A quantidade de estudantes do gênero feminino e masculino foi equilibrada, com um maior percentual de indivíduos do gênero feminino (59\%) em relação ao masculino (41\%). Na divisão de gênero entre os períodos (Figura 1B), houve uma maior participação do feminino nos quatro períodos $\left(67 \%\right.$ no $2^{\circ}, 53 \%$ no $3^{\circ}, 55 \%$ no $4^{\circ}$ e $61 \%$ no $6^{\circ}$ período), com um quantitativo menor apenas no último ( $33 \%$ no $10^{\circ}$ período).

Devido a maior quantidade de alunos ingressantes ( $2^{\circ}$ período), a faixa etária predominante foi de acadêmicos de 20 a 24 anos, correspondendo a $53 \%$ da amostra, e que haviam cursado apenas uma disciplina de Química experimental, Química Geral (Figura 1C e 1D). Em seguida, os participantes veteranos que haviam cursado 4 ou mais disciplinas de Química experimental (28\%) e que apresentavam idade acima de 25 anos.

\section{Detecção da dificuldade}

Deu-se prosseguimento ao objetivo de detectar as dificuldades dos discentes referentes aos tópicos que norteiam a elaboração de um relatório de prática experimental. Para tanto, utilizou-se o gráfico treemap para apresentar as principais dificuldades relacionadas aos itens com maior dificuldade e os com menor dificuldade. Gráficos do tipo diagrama de árvore (treemap) são ferramentas estatísticas utilizadas para visualizar dados de uma forma hierárquica, utilizando dados agrupados que refletem as categorias utilizadas e a intensidade por meio de retângulos dimensionados diferentemente, conforme os valores numéricos para cada ramificação. Quanto maior o retângulo, maior o valor numérico. Por meio do gráfico treemap foi possível identificar que os itens "justificativa, objetivos e conclusão", foram as categorias que apresentam uma menor intensidade, o que demonstra pouca dificuldade dos acadêmicos para os referidos itens.

Entre as categorias utilizadas no gráfico, citam-se o objetivo, a introdução, a justificativa, o referencial teórico, a metodologia, os resultados e discussão, a conclusão, as referências bibliográficas e a formatação nas normas. Os resultados podem ser visualizados na Figura 2, e observados na Tabela 1.

Os itens com maiores valores numéricos, refletindo as categorias que apresentaram maior dificuldade, foram: "Resultados e discussão," "formatação nas normas" e "referencial teórico", sendo o primeiro, o item preponderante em todos os períodos, com $43 \%$ no $2^{\circ} ; 53 \%$ no $4^{\text {o }} ; 70 \%$ no $6^{\circ} ; 69 \%$ no $8^{\circ}$ e $100 \%$ no $10^{\circ}$.

De acordo com Squier, Renaud e Larsen, ${ }^{17}$ as disciplinas de Química experimentais apresentam um viés completo de comunicação científica, podendo ser trabalhado tanto a escrita científica, quanto as habilidades de comunicação oral, por meio de seminários com enfoque científico nas reações e transformação da matéria. Por tratar-se de disciplinas classificadas como "exatas", as químicas experimentais comumente focam no conteúdo e nos fenômenos 

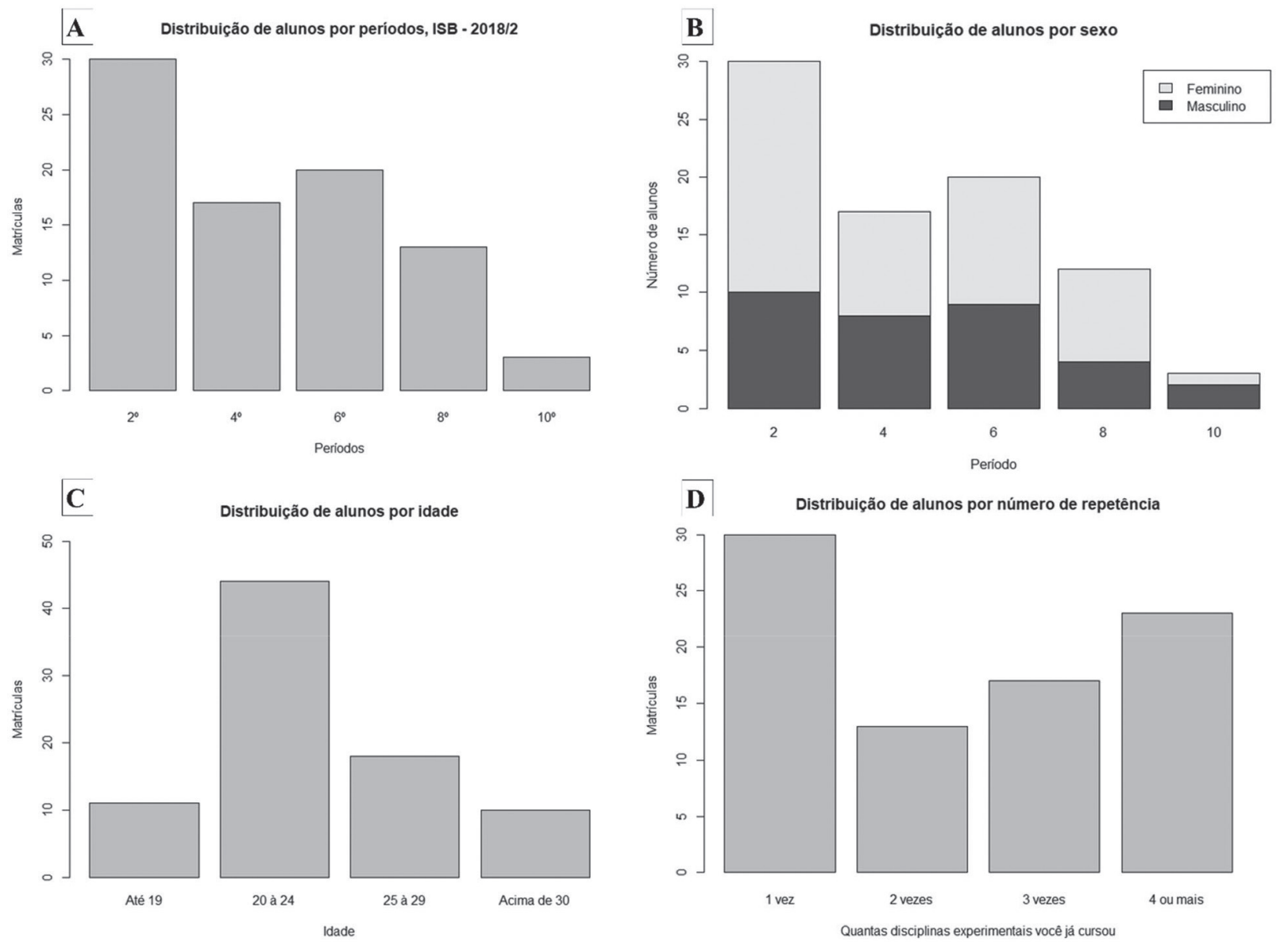

Figura 1. Caracterização dos sujeitos (Fonte: Dados Primários, 2019)

Detecção da dificuldade na elaboração de relatórios científicos
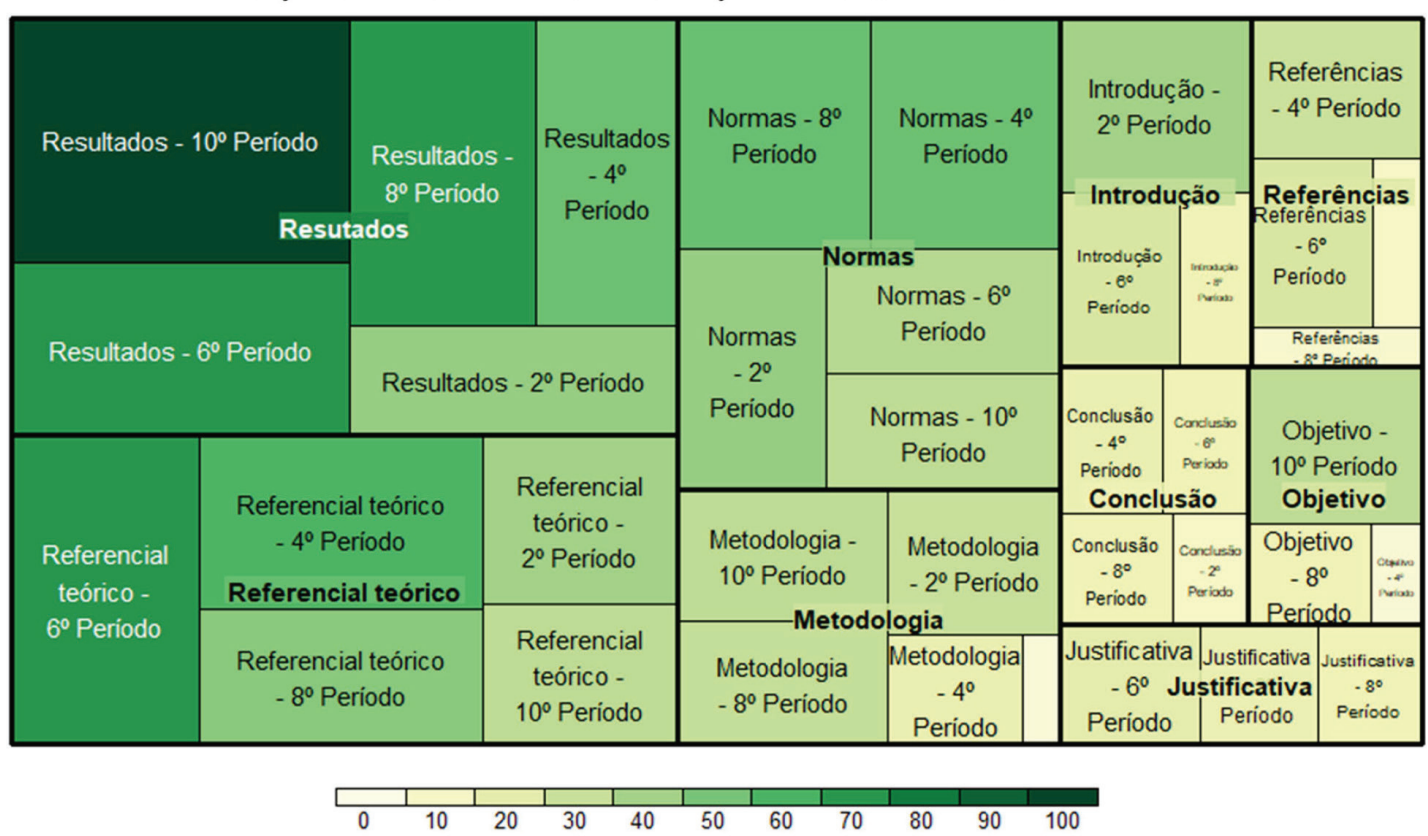

Figura 2. Detecção da dificuldade na elaboração de relatórios científicos (Fonte: Dados Primários, 2019)

coletados no laboratório para elaboração do material escrito e, os aspectos didáticos acabam sendo negligenciados.

Os dados detectados nessa pesquisa corroboram com as pesquisas descritas na literatura sobre as questões de estrutura e linguagem dos textos em relatórios de atividades experimentais, em que nem sempre estas são objeto de análise cuidadosa por parte dos docentes que no geral, apenas valorizam a observação dos conteúdos apresentados. Na revisão de Oliveira e Queiroz, ${ }^{5}$ os autores citam que, embora as disciplinas de Ciências/Química disponibilizem dados utilizáveis na produção de trabalhos dos estudantes, nem sempre as questões de 
Tabela 1. Detecção da dificuldade na elaboração de relatórios científicos

\begin{tabular}{|c|c|c|c|c|c|c|c|c|c|c|}
\hline \multirow{2}{*}{$\begin{array}{l}\text { Item com dificuldade } \\
\text { Período }\end{array}$} & \multicolumn{5}{|c|}{ Quantidade por período $(\mathrm{N})$} & \multicolumn{5}{|c|}{ Quantidade por período (\%) } \\
\hline & $2^{\circ}$ & $4^{\circ}$ & $6^{\circ}$ & $8^{\circ}$ & $10^{\circ}$ & $2^{\circ}$ & $4^{\circ}$ & $6^{\circ}$ & $8^{\circ}$ & $10^{\circ}$ \\
\hline Objetivos & 0 & 1 & 0 & 2 & 1 & 0 & 6 & 0 & 15 & 33 \\
\hline Introdução & 12 & 0 & 5 & 2 & 0 & 40 & 0 & 25 & 15 & 0 \\
\hline Justificativa & 5 & 0 & 4 & 2 & 0 & 17 & 0 & 20 & 15 & 0 \\
\hline Referencial teórico & 12 & 10 & 14 & 6 & 1 & 40 & 59 & 70 & 46 & 33 \\
\hline Metodologia & 9 & 3 & 1 & 4 & 1 & 30 & 18 & 5 & 31 & 33 \\
\hline Resultados e discussão & 13 & 9 & 14 & 9 & 3 & 43 & 53 & 70 & 69 & 100 \\
\hline Conclusão & 3 & 3 & 3 & 2 & 0 & 10 & 18 & 15 & 15 & 0 \\
\hline Referências bibliográficas & 3 & 5 & 5 & 1 & 0 & 10 & 29 & 25 & 8 & 0 \\
\hline Formatação nas normas & 13 & 9 & 7 & 7 & 1 & 43 & 53 & 35 & 54 & 33 \\
\hline
\end{tabular}

estrutura e linguagem do texto são objeto de análise cuidadosa por parte do docente. Dessa forma, mesmo quando os docentes solicitam dos estudantes a produção de textos científicos, a análise e avaliação nem sempre consegue contemplar a escrita científica de forma plena, resumindo-se na observação dos conteúdos apresentados e aspectos superficiais de organização do texto. Tal análise é reportada por pesquisadores em outros artigos publicados com essa temática e são corroboradas por pesquisas internacionais sobre a comunicação científica no ensino de Ciências. ${ }^{5,9,18,19}$

Sabe-se que os cursos universitários apresentam disciplinas específicas sobre metodologia de trabalho científico e Português instrumental, como é o caso do curso dos sujeitos investigados, no entanto, essas disciplinas são ministradas nos períodos iniciais da graduação, bem como Informática básica, em que são trabalhados os itens formatação de trabalhos científicos. No entanto, o que foi relatado informalmente pelos acadêmicos foi a pouca interdisciplinaridade visando a aplicação das abordagens sobre comunicação científica e formatação nos conteúdos de Química, tendo como consequência conhecimentos isolados sobre os assuntos.

Ao serem questionados sobre o meio em que costumam utilizar para pesquisar assuntos para o relatório experimental (questão a), o percentual médio foi de artigos (65\%), seguido de livros (59\%) (Tabela 2). As questões “A, B, C e E” da Tabela 2 eram questões objetivas, no entanto, que poderiam ter mais de uma opção. Para análise dos dados foi contabilizado a quantidade de seleções e avaliado o quantitativo de discentes do período participantes da pesquisa.

Tabela 2. Análise da dificuldade dos discentes na elaboração de relatórios experimentais

\begin{tabular}{|c|c|c|c|c|c|c|}
\hline \multirow{2}{*}{$\begin{array}{l}\text { Perguntas } \\
\text { Período letivo } \\
\end{array}$} & \multirow[t]{2}{*}{ Opções } & \multicolumn{5}{|c|}{ Respostas $(\%)$} \\
\hline & & 2 & 4 & 6 & 8 & 10 \\
\hline \multirow{5}{*}{$\begin{array}{l}\text { a) Em qual material você } \\
\text { costuma pesquisar assuntos para } \\
\text { elaboração do relatório? }\end{array}$} & Artigos & 63 & 53 & 60 & 85 & 33 \\
\hline & Revista & 0 & 0 & 0 & 15 & 0 \\
\hline & Livros & 40 & 53 & 70 & 61 & 100 \\
\hline & Resumos & 13 & 12 & 0 & 0 & 33 \\
\hline & Outros & 7 & 18 & 65 & 23 & 0 \\
\hline \multirow{5}{*}{$\begin{array}{l}\text { b) Em que local você costuma } \\
\text { pesquisar para elaboração do } \\
\text { relatório? }\end{array}$} & Biblioteca & 40 & 35 & 60 & 46 & 67 \\
\hline & Laboratório de informática & 33 & 29 & 50 & 31 & 33 \\
\hline & Em casa & 33 & 29 & 25 & 46 & 33 \\
\hline & Residência de alguém com internet & 40 & 29 & 25 & 23 & 67 \\
\hline & Outros & 7 & 12 & 5 & 31 & 33 \\
\hline \multirow{6}{*}{$\begin{array}{l}\text { c) Qual a dificuldade que você } \\
\text { encontra na elaboração de } \\
\text { relatórios experimentais }\end{array}$} & Poucos livros disponíveis & 27 & 41 & 25 & 38 & 67 \\
\hline & Dificuldade em acessar a Internet & 50 & 71 & 65 & 69 & 67 \\
\hline & Linguagem complexa dos artigos e livros & 23 & 29 & 30 & 38 & 0 \\
\hline & Não sei procurar as referências & 7 & 12 & 5 & 8 & 33 \\
\hline & $\begin{array}{l}\text { Ausência ou quantidade insuficiente de orientação dos } \\
\text { docentes }\end{array}$ & 27 & 35 & 10 & 15 & 0 \\
\hline & Alta quantidade de disciplinas & 27 & 29 & 25 & 23 & 33 \\
\hline \multirow{2}{*}{$\begin{array}{l}\text { d) Você tem hábito de ler } \\
\text { trabalhos científicos }\end{array}$} & Sim & 47 & 42 & 63 & 54 & 67 \\
\hline & Não & 53 & 58 & 37 & 38 & 33 \\
\hline \multirow{5}{*}{ e) Se sim, qual? } & Artigos & 57 & 80 & 75 & 86 & 100 \\
\hline & Revista & 7 & 20 & 0 & 0 & 50 \\
\hline & Livros & 36 & 0 & 25 & 43 & 50 \\
\hline & Resumos & 21 & 0 & 8 & 0 & 0 \\
\hline & Outros & 14 & 0 & 8 & 0 & 0 \\
\hline \multirow{4}{*}{ f) Qual a frequência média? } & Semestral & 29 & 0 & 25 & 29 & 100 \\
\hline & Mensal & 50 & 60 & 50 & 71 & 0 \\
\hline & Quinzenal & 7 & 0 & 8 & 0 & 0 \\
\hline & Semanal & 21 & 20 & 25 & 29 & 0 \\
\hline
\end{tabular}


O uso de artigos científicos nas disciplinas é uma tendência que está acontecendo nos cursos de graduação e vem sendo incentivada por docentes em âmbito nacional e internacional. Segundo as pesquisas, o uso de artigos facilita a compreensão dos acadêmicos em relação as particularidades de uma pesquisa científica e mesmo que apresente alguns pontos de diferença com os trabalhos acadêmicos, familiariza os estudantes aos termos em comum, além de situá-los nas pesquisas científicas atuais e clássicas. ${ }^{14}$

Aproximadamente metade dos acadêmicos relataram que o local físico utilizado para elaboração do relatório é a biblioteca, seguido de locais com acesso à internet (37\%). Esse resultado corrobora com o detectado pela questão seguinte, na qual foram analisados os principais pontos que dificultam a elaboração do relatório, em que $65 \%$ afirmaram que a principal limitação é relacionada ao uso de internet seguida da pouca disponibilidade dos livros (39\%).

Esse é um item pouco detectado nas pesquisas em outras universidades, mas um fator que merece destaque nesse trabalho. A UFAM apresenta diferentes polos fora da sede que se localizam na capital. O ISB é um campus situado no município de Coari, 363 km de Manaus, e apresenta os rios como principal meio de transporte. $\mathrm{O}$ acesso à internet no período da pesquisa realizada não era contemplado de forma satisfatória aos estudantes, ocorrendo somente por via de rádio, sem a presença de alternativas como fibra óptica. No Instituto há dois laboratórios de informática para atender os universitários, no entanto, eles não suprem a demanda dos estudantes devido à baixa velocidade da Internet, o que reflete na dificuldade reportada pelos discentes.

Segundo Yamaguchi e Furtado, ${ }^{10}$ a leitura passa para além do mundo virtual, realizada também em material bibliográfico impresso, no entanto, a Internet é um veículo de maior fluidez na divulgação de trabalhos científicos, relevante para o desenvolvimento humano e contribui consideravelmente nas pesquisas referenciais.

$\mathrm{Na}$ análise do hábito de leitura (questão d), 55\% dos estudantes declararam ter o hábito de ler e destes, $79 \%$ afirmaram que o artigo é o principal instrumento. Avaliando os períodos, os acadêmicos mais antigos apresentaram maior percentual de leitores, principalmente do $6^{\circ}$ ao $10^{\circ}$ período. Em contrapartida, a frequência de leitura declarada pelo público analisado foi mensal, correspondendo a $58 \%$ dos entrevistados.

O hábito de leitura perpassa a história das Ciências e está diretamente ligada à aprendizagem significativa. Assim, para a elaboração de um material que reflita o aprendizado de forma satisfatória, é necessário além da informação adquirida, uma familiarização com os princípios científicos e a visão ampliada do que se pretende expor.

Assim, a leitura de teorias e trabalhos científicos é indispensável para o andamento de um relatório experimental, tal qual preconizam as Diretrizes Curriculares Nacionais que apontam para a necessidade dos estudantes de Química aprenderem não somente os princípios da Ciência, envolvendo os conceitos, leis e princípios da química, mas ir além, contemplando também a leitura, compreensão e interpretação dos textos científico-tecnológicos. Além de saber defender seus achados, é necessário que os acadêmicos se apropriem da escrita e leitura para apresentar e comunicar corretamente os projetos e resultados científicos. Esse conhecimento utilizado é obtido ao longo da sua vida e, sem esse, dificilmente haverá compreensão plena dos fenômenos existentes e uma aprendizagem significativa. ${ }^{20}$

A dificuldade dos discentes reflete no percentual de retenção das disciplinas, conforme pode ser observado na Figura 3, por meio do índice de retenção dos discentes nas disciplinas experimentais ao longo dos 12 anos do Instituo de Saúde e Biotecnologia, no curso de Ciências: Biologia e Química.

Em relação às retenções nas disciplinas experimentais, verificou-se que a média das retenções durante os 12 anos foi de 20,48 $\pm 0,18$, sendo que Química Inorgânica apresentou o maior índice com $21 \%$, seguido de Química Geral (21\%), Analítica (20\%) e Físico-Química (20\%). Os índices de retenção podem demonstrar a dificuldade que os discentes têm devido à falta de comunicação entre o que eles aprendem e a forma como podem expressar esse aprendizado.

\section{Intervenção}

Frente a importância da comunicação científica, desenvolver ações voltadas para a busca de atuações que possam promover a redução dessas dificuldades, ou mesmo, a eliminação, torna-se essencial. Na perspectiva dos discentes, ações para auxiliar a elaboração dos relatórios seria o uso de apostilas ou manuais de elaboração de relatórios $(41 \%)$, seguidos de cursos ou oficinas $(32 \%)$ e aulas de elaboração durante a disciplina $(25 \%)$. As respostas por categorias podem ser visualizadas na Figura 4.

Estratégias para auxiliar os estudantes de graduação a desenvolverem habilidades de redação técnica em relatórios experimentais estão sendo descritas nas universidades públicas e privadas visando contribuir com o desenvolvimento de táticas eficientes para auxiliar na escrita desse gênero.

A utilização de artigos de periódicos e textos científicos têm o objetivo de fornecer aos alunos uma introdução às técnicas necessárias para uma redação técnica. A metodologia investigativa descrita por Cacciatore e Sevian ${ }^{21}$ utilizando como tema gerador a aplicação de Química Verde em uma abordagem investigativa facilitou a compreensão de acadêmicos sobre a importância e as qualidades essenciais da comunicação escrita dos artigos e relatórios acadêmicos. Nesse

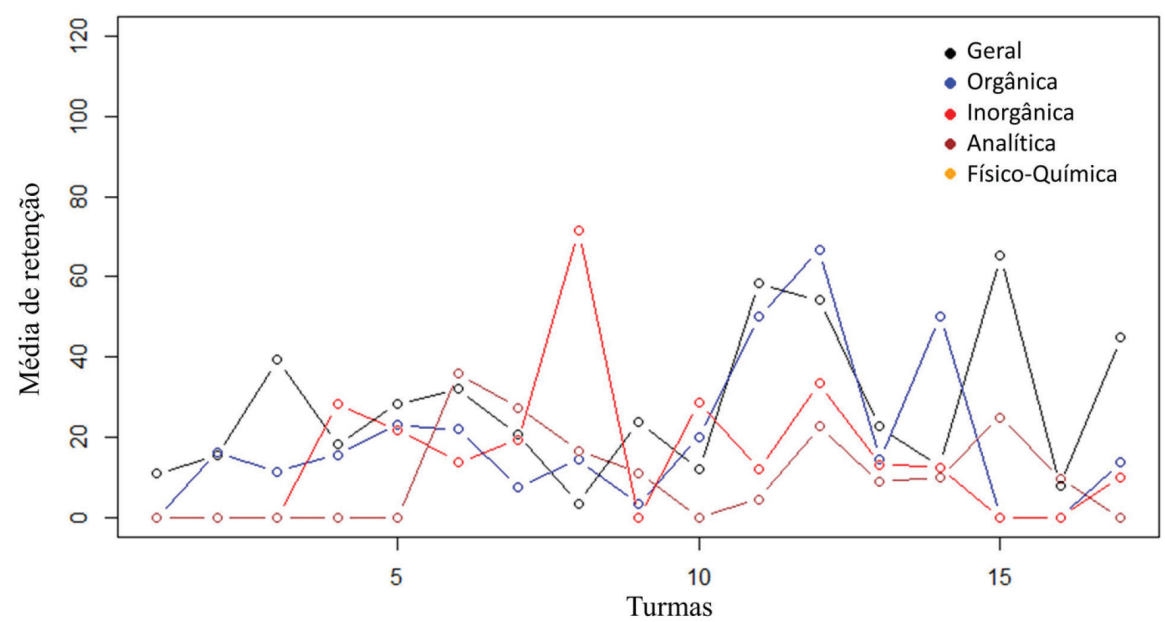

Figura 3. Índice de retenção em Química experimental (Fonte: Dados Primários, 2019) 


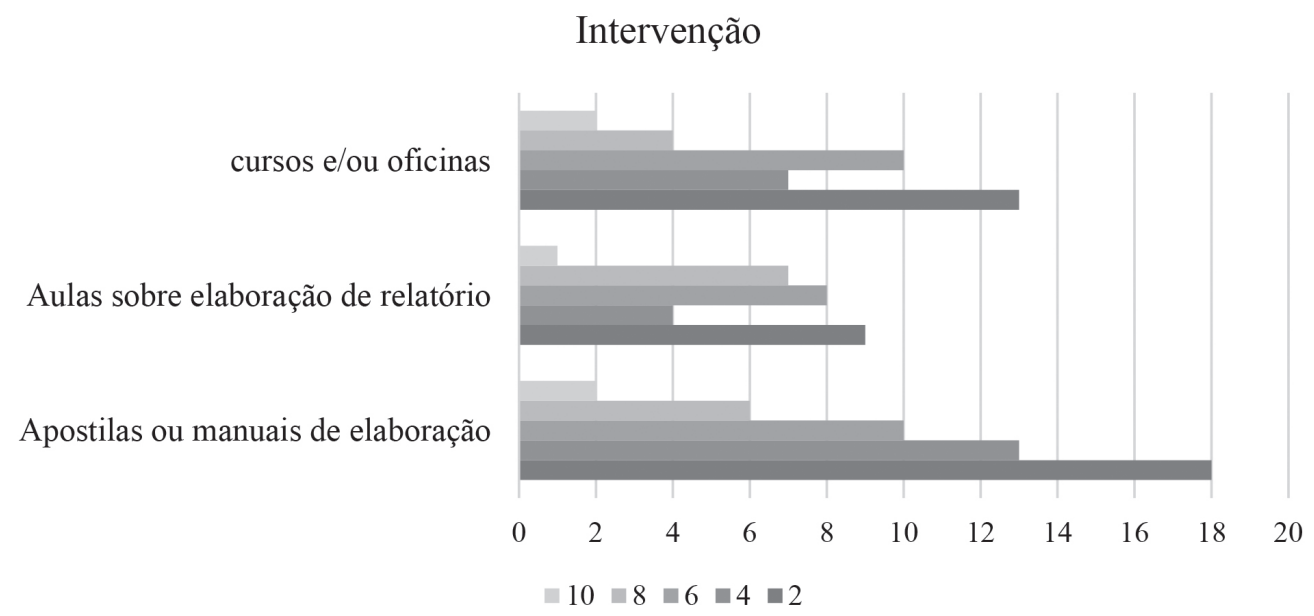

Figura 4. Sugestões para auxiliar na elaboração de relatórios experimentais (Fonte: Dados Primários, 2019)

trabalho, os autores apresentaram a importância da qualidade das informações para que as atividades práticas pudessem ser realizadas. Os acadêmicos vivenciaram as dificuldades de realizarem um procedimento experimental com a ausência de dados e puderam verificar a importância da padronização em uma comunicação científica.

O uso de trabalhos científicos em discussões sobre o desenvolvimento dos fenômenos que foram observados ou que serão investigados vêm como uma ferramenta pedagógica que visa auxiliar no entendimento dos fenômenos e na construção do conhecimento dos discentes, possibilitando argumentações sobre os resultados baseados na leitura e conhecimentos teóricos prévios. ${ }^{14}$

Por meio dos resultados detectados e corroborando com os achados na literatura, verifica-se que uma das principais formas de contribuir com o desenvolvimento dos estudantes na escrita científica dar-se-á pelo incentivo à leitura, ampliando os conhecimentos e colaborando com uma visão ampla dos usos da linguagem científica. A importância de se integrar a leitura e a escrita no ensino de química é uma forma de promover tanto a aprendizagem quanto o desenvolvimento de habilidades de comunicação, análise crítica e argumentação. ${ }^{22}$

Assim, a melhoria da qualidade dos relatórios experimentais torna-se uma premissa para o aprimoramento da qualidade do aprendizado dos acadêmicos, e a participação dos professores como motivadores é de suma importância para que isso aconteça. Torna-se necessário que os docentes possam mediar o processo de desenvolvimento intelectual dos discentes, utilizando estratégias que minimizem as dificuldades dos acadêmicos, orientando para a busca do conhecimento científico e profissional.

\section{CONCLUSÃO}

A dificuldade na elaboração de relatórios experimentais relacionou-se à dificuldade na apropriação da linguagem científica. Este trabalho contribui com as discussões sobre a temática proposta, apresentando que a dificuldade se relaciona principalmente com a falta de leitura e, consequentemente, pouca interpretação dos dados. Os discentes conseguem entender os fenômenos, mas apresentam dificuldade em demonstrá-los por meio da escrita científica. Dessa forma, os procedimentos de intervenção devem contemplar desde os períodos iniciais até o final da graduação, a fim de que os discentes possam conseguir demonstrar de forma satisfatória o aprendizado adquirido e desenvolver-se como profissionais.

Os resultados demonstraram que a principal dificuldade se relaciona a itens que exigem leitura e conhecimento prévio. Nesse contexto, o docente apresenta-se como protagonista no papel de orientar os discentes sobre os caminhos que eles podem seguir, seja por meio de indicação de materiais didáticos, uso de artigos científicos, materiais complementares e o acompanhamento na disciplina. Espera-se com os resultados dessa pesquisa contribuir com os estudos existentes sobre as formas de intervenção que visem auxiliar os discentes na elaboração de relatórios experimentais, criando subsídios que favoreçam estudos mais aprofundados sobre o tema, melhorando assim o nível de educação e desenvolvimento em nosso país.

\section{REFERÊNCIAS}

1. Luz Jr., G. E.; Sousa, S. A. A.; Moita, G. C.; Moita Neto, J. M.; Quim. Nova 2004, 27, 164.

2. Galiazzi, M. do C.; Gonçalves, F. P.; Quim. Nova 2004, 27, 326.

3. Lima, I. M.; Moraes, M. L.; Sousa, D. P.; Barros, J. C.; Pessoa, P. A. P.; $V$ Congresso Nacional de Educação 2017, 1.

4. Shiland, T. W.; Shiland, T. W.; J. Chem. Educ. 1999, 76, 108.

5. Oliveira, J. R. S.; Queiroz, S. L.; Quim. Nova 2015, 38, 553.

6. Oliveira, J. R. S.; Queiroz, S. L.; Quim. Nova 2008, 31, 1263.

7. Bessa, J. C. R.; Bernardino, R. A. dos S.; Nascimento, I. A. de A.; Encontros de Vista 2005, 10, 1.

8. Motta, I. L. O. M.; Revista Científica Eletrônica de Ciências Sociais Aplicadas da Eduvale 2010, 5, 1.

9. Oliveira, J. R. S.; Batista, A. A.; Quim. Nova 2010, 33, 1980.

10. Yamaguchi, K. K. de L.; Furtado, M. A. S.; Educação Online 2018, 1, 108.

11. Oliveira Jr., O. N.; Rev. Bras. Ensino Física 2015, 37, 2201.

12. Rocha, J. S.; Vasconcelos, T. C.; XVIII Encontro Nac. Ensino Química (XVIII ENEQ) 2016, Florianópolis, Brasil.

13. Han, N. S.; Li, H. K.; Sin, L. C.; Sin, K. P.; Educational Science 2011 , 2,45 .

14. Tilstra, L.; J. Chem. Educ. 2001, 78, 762.

15. Henary, M.; Owens, E. A.; Tawney, J. G. J.; Chem. Educ. 2015, 92, 90.

16. Gil, A. C.; Em Como elaborar projetos de pesquisa, $4^{\mathrm{a}}$ ed., Editora Atlas: São Paulo, 2008.

17. Squier, C.; Renaud, J.; Larsen, S.; J. Chem. Educ. 2006, 87, 1029.

18. Forest, K.; Rayne, S.; J. Chem. Educ. 2009, 86, 1290.

19. Oliveira, J. R. S.; Queiroz, S. L.; Educação 2012, 35, 851.

20. Lerner, N.; Written Communication 2007, 24, 191.

21. Cacciatore, K. L.; Sevian, H.; J. Chem. Educ. 2006, 83, 1039.

22. Francisco Jr., W. E.; Garcia Júnior, O.; Quim. Nova Esc. 2010, 32, 191. 